\title{
INNOVACIÓN EMPRESARIAL: UNA MIRADA DESDELA COMPETITIVIDAD, EL DESARROLLO LOCAL Y LATRANSFORMACIÓN PRODUCTIVA PARA LA INTERNACIONALIZACIÓN EN COLOMBIA*
}

Recibido: 09 de mayo de 2013 - Aprobado: 22 de octubre de 2013

\author{
Marleny Cardona Acevedo** \\ Suelen Castiblanco Moreno*** \\ Hugo Díaz Sánchez ${ }^{* * *}$
}

\section{RESUMEN}

Este artículo analiza la innovación en las pequeñas y medianas empresas importadoras y exportadoras de Bogotá desde el desarrollo local, la competitividad y la transformación productiva. Se contrasta la información obtenida en entrevistas semiestructuradas con las conceptualizaciones de la revisión teórica. Los empresarios entrevistados coinciden en que la innovación va más allá de sus esfuerzos individuales. Resulta de acciones desde el territorio que estimulan desarrollo local, dinámicas de competitividad e internacionalizaciones exitosas. Aunque reconocen que las alianzas productivas permiten economías de escala y avances desde adentro hacia fuera, son reacios a participar en ellas pues faltan garantías para compartir las innovaciones propias.

\section{PALABRAS CLAVE}

Servicios, transformación productiva, innovación, competitividad, desarrollo local e internacionalización.

\section{CLASIFICACIÓN JEL}

L84, M16, O31, R58.

\section{CONTENIDO}

1. Introducción. 2. Antecedentes: innovación en la competitividad, la transformación productiva y el desarrollo local. 3. Resultados. 4. Discusión; Bibliografía.

Artículo de reflexión. Forma parte de la investigación "Internacionalización de la innovación en las empresas colombianas desde la competitividad, el desarrollo local y la transformación productiva", adscrita al grupo de investigación "Desarrollo local, competitividad y transformación productiva" y realizada en el año 2012 con financiamiento de la Fundación Universitaria Konrad Lorenz.

** Doctora en Niñez y Juventud del Centro de Estudios Avanzados en Niñez y Juventud (CINDE) Universidad de Manizales, Manizales, Colombia. Doctora en Ciencias Sociales del Colegio de la Frontera Norte, Tijuana, México. Economista Universidad de Antioquia, Medellín, Colombia. Directora Centro de Investigaciones, Escuela de Negocios, Fundación Universitaria Konrad Lorenz, Bogotá, Colombia. Miembro del Grupo de investigación Desarrollo local, competitividad y transformación productiva. Correo electrónico: marleny.cardona@konradlorenz.edu.co.

.*. Estudiante de maestría en Estudios Interdisciplinarios en desarrollo, Universidad de los Andes, Bogotá, Colombia. Economista, Universidad de la Salle, Bogotá, Colombia. Docente e investigadora, Escuela de Negocios, Fundación Universitaria Konrad Lorenz, Bogotá, Colombia. Miembro del Grupo de investigación Desarrollo local, competitividad y transformación productiva. Correo electrónico: suelene.castiblancom@konradlorenz.edu.co.

...* Magíster en Relaciones Internacionales, Pontificia Universidad Javeriana y Escuela de Negocios de la Fundación Universitaria Konrad Lorenz, Bogotá, Colombia. Miembro del Grupo de investigación Desarrollo local, competitividad y transformación productiva. Correo electrónico: hugo.diaz@konradlorenz.edu.co. 


\section{CORPORATE INNOVATION: A VIEW FROM COMPETITIVENESS, LOCAL DEVELOPMENT AND PRODUCTIVE TRANSFORMATION FOR THE INTERNATIONALIZATION OF COLOMBIA}

\section{ABSTRACT}

This article analyzes innovation on small and medium import-export companies of Bogota from local development, competitiveness and productive transformation. A comparison is made between the information obtained on semi structured interviews and the conceptualization of the theoretical review. The interviewed businessmen agree on the fact that innovation is larger than individual efforts. It is the result of actions taken from the territory that stimulate local development, dynamics of competitiveness and successful internationalizations. Even though they also recognize that productive alliances allow economies of scale and inside out driven advancements, they are reluctant to participate in them due to the lack of guarantees in shearing their in-hose innovations.

\section{KEY WORDS}

Productive transformation services, innovation, competitiveness, local development and internationalization.

\section{JEL CLASSIFICATION}

L84, M16, O31, R58.

\section{CONTENT}

1. Introduction, 2. Background: Innovation in competitiveness, productive transformation and local development. 3. Results. 4. Discussion, Bibliography.

\section{INOVAÇÃO EMPRESARIAL:UMAMIRADA DESDE A COMPETITIVIDADE, ODESENVOLVIMENTO LOCALE A TRANSFORMAÇÃO PRODUTIVA PARA A INTERNACIONALIZAÇÃO NA COLÔMBIA RESUMO}

Este artigo analisa a inovação nas pequenas e médias empresas importadoras e exportadoras de Bogotá desde o desenvolvimento local, a competitividade e a transformação produtiva. Contrasta-se a informação obtida em entrevistas semiestruturadas com as conceptualizações da revisão teórica. Os empresários entrevistados coincidem em que a inovação vai além de seus esforços individuais. Resulta de ações desde o território que estimulam desenvolvimento local, dinâmicas de competitividade e internacionalizações exitosas. Ainda que reconhecem que as alianças produtivas permitem economias de escala e avanços desde adentro para fora, são arredios a participar nelas pois faltam garantias para compartilhar as inovações próprias.

\section{PALAVRAS CHAVES}

Serviços, transformação produtiva, inovação, competitividade, desenvolvimento local e internacionalização.

\section{CLASSIFICAÇÃO JEL}

L84, M16, Ou31, R58.

\section{CONTEÚDO}

1. Introdução. 2. Antecedentes: inovação na competitividade, a transformação produtiva e o desenvolvimento local. 3. Resultados. 4. Discussão; Bibliografia. 


\section{INTRODUCCIÓN}

La innovación es una preocupación constante en las organizaciones, por sus efectos deseables en los mercados nacionales y extranjeros. La innovación, como expresión del desarrollo, es un proceso resultado de la acumulación de conocimiento desde el territorio, y como tal crece en la medida en que las empresas participan de la apertura a la competencia extranjera.

Asimismo, la innovación depende de la identificación de los factores determinantes del desarrollo tecnológico, del conocimiento de las características de los procesos locales y del estado del desarrollo y avance tecnológico en el país, desde la construcción de políticas públicas. A través de estas, se promueve la innovación social e individual que crea condiciones para un posicionamiento de las unidades productivas, cada vez más maduro, con competitividad y transformación productiva.

Si bien la interrelación entre las condiciones que permiten el liderazgo sostenido en los mercados y la internacionalización ha sido estudiada ampliamente (Cerrato $\varepsilon$ Depperu, 2011), los impactos que los programas de reconversión industrial y la dimensión territorial del desarrollo tienen en estos procesos están aún por estudiarse. Poder establecer correlaciones entre estas dimensiones permitiría una mejor formulación y evaluación de políticas públicas, a la vez que ayudaría a comprender mejor la relación entre internacionalización e innovación.

Este artículo constituye un avance en el análisis de la relación entre la innovación, el desarrollo local, la competitividad y la transformación productiva y su papel en la internacionalización. En este sentido, en el primer aparte se presenta una revisión teórica sobre la relación entre las dimensiones objeto de interés; en el segundo aparte se incorpora a esta discusión la categoría de internacionalización; en el tercer aparte se presentan los resultados del trabajo empírico realizado con ocho pymes importadoras y exportadoras de la ciudad de Bogotá. Las empresas entrevistadas tienen capital $100 \%$ nacional, con excepción de una de ellas, y fueron creadas hace menos de 10 años; pertenecen a los sectores manufacturero, alimentos e importaciones; tienen más de diez trabajadores y el capital es nacional. Además, son producto del ahorro familiar, en su mayoría.

Finalmente, se presenta una discusión sobre el rol de las dimensiones en la innovación. La innovación es una dimensión que recoge el desarrollo local como recurso, la transformación productiva como medio, y la competitividad como objetivo. El territorio es espacio de interacción de las mismas que hace posible su causación circular. 


\section{ANTECEDENTES: INNOVACIÓN EN EL DESARROLLO LOCAL, LA TRANSFORMACIÓN PRODUCTIVA Y LA COMPETITIVIDAD}

El territorio como espacio de desarrollo, permite la combinación del capital humano y los recursos naturales que fortalecen el tejido social posibilitando la transformación productiva y la competitividad. Es en este espacio donde la innovación colectiva e individual se materializa a través del aprendizaje local. De acuerdo con el manual de Oslo (Organización para la Cooperación y el Desarrollo Económico OCDE, 2005), citado por Robledo, Gómez y Restrepo $(2008$, p. 2) la innovación puede ser definida como "La implementación de un producto, proceso, método organizacional o método de mercadeo nuevo o significativamente mejorado".

Las primeras aproximaciones a la innovación se hicieron bajo un enfoque microeconómico, centrado en las características de las empresas innovadoras, así estos estudios, de corte schumpeteriano, asumen que en las empresas se presentan ciclos de innovaciones radicales, que luego se expanden al resto de la industria y se correlacionan con los ciclos de auge económico (Méndez, 2002).

Por otro lado, el surgimiento de un enfoque más macroeconómico hizo que la teoría del crecimiento endógeno y los estudios urbanos y regionales centraran sus análisis en las posibilidades que ofrecen los territorios para la localización de la innovación y el desarrollo (IED), la producción de la tecnología y el rol de las interacciones espaciales, expresadas a través de redes de innovadores, complejos regionales de innovación e infraestructura regional del conocimiento (Acs, 2002).

La posterior incorporación a estos estudios de los aportes tempranos sobre la importancia de la relación entre geografía e industria, hicieron que la innovación se concibiera de forma más amplia y se incorporaran a su análisis las características específicas de los lugares en los que se produce, dando origen a los estudios sobre distritos industriales, clusters, sistemas regionales de innovación, entre otros (Méndez, 2002).

Finalmente, en los últimos años, la denominada "economía del conocimiento" ha puesto su atención en la capacidad de los territorios para utilizar el conocimiento y aprendizaje colectivo como recursos específicos; aquí se ubican conceptos como las regiones inteligentes o territorios que aprenden. Es en el marco de este último enfoque en el que la relación entre transformación productiva, desarrollo local y competitividad con miras a la innovación, es más fuerte (Méndez, 2002). 
Innovación empresarial: una mirada desde la competitividad, el desarrollo local y la transformación productiva ...

En esta investigación se suponen dos elementos clave para el impulso de la innovación en los territorios: las interacciones espaciales y el capital humano.

Dahlman y Frischtak (1993, p. 414) definen los sistemas de innovación como "la red de agentes y conjunto de políticas e instituciones que afectan la introducción de tecnología que es nueva en la economía". Para Vásquez Barquero (2009), y Boisier y Canzanelli (2009) es la acción coordinada de estos agentes, es decir, el trabajo de los sistemas de innovación, la que permite mejorar la productividad, competitividad y, de forma más amplia, el desarrollo de los territorios. Esto, debido a que las empresas transforman el ahorro en inversión, y el estímulo a redes y agrupaciones entre entidades, propicia la aparición de economías de escala, de proximidad y reduce los costos de transacción.

Por el lado del capital humano, la capacitación provee condiciones internas para crear innovación y aceptar la que proviene del exterior. Empleados con mayores niveles de educación formal e informal ven en el avance tecnológico una herramienta para hacer más productivo su trabajo; por el contrario, los empleados con baja capacitación ven la innovación como una amenaza para sus ocupaciones y la rechazan. La capacitación no es solo punto de partida de la innovación sino fin de los procesos de transformación de los territorios y una de las manifestaciones aplicadas del impacto positivo de la competitividad y productividad en la vida de las personas.

Es por esto que Robledo et al. (2008) afirman que detrás de la innovación se encuentran las capacidades organizacionales como promotoras de nuevas dinámicas. Sugieren que el término "capacidades de innovación tecnológica" (CIT) agrupe aquellas capacidades organizacionales relacionadas con los procesos que se dan en la empresa para producir innovación como resultado de la gestión estratégica u operativa (Cardona y Escobar, 2012). De forma particular, se citan dos de las capacidades ligadas al desarrollo local mediante la administración del capital humano. Estas capacidades son: la capacidad de gestión de recursos y la capacidad de aprendizaje organizacional.

La capacidad de gestión de recursos hace referencia a la habilidad que tienen las empresas para adquirir y asignar de manera apropiada experiencia y tecnología a los procesos de innovación (Acs \& Amorós, 2008). Es decir, es la capacidad que tienen las firmas para hacer una adecuada gestión de la capacitación formal e informal, en el fortalecimiento de perfiles en funciones generales y específicas, capacitación que es recibida por los trabajadores a lo largo de toda su trayectoria laboral. 
La capacidad de aprendizaje organizacional es la habilidad para identificar, asimilar y aprovechar el conocimiento proveniente del exterior. Robledo et al. (2008) la definen como la habilidad de mantener o mejorar el desempeño basado en la experiencia, y hace referencia al 'learning by doing'. Esta capacidad posibilita la adquisición del conocimiento implícito y explícito, el traspaso y el uso del conocimiento.

Estas capacidades están vinculadas con el desempeño innovador y económico de las organizaciones y, por lo tanto, con la transformación productiva. Esta se garantiza en los procesos, acciones y políticas establecidas para su desarrollo con incorporación de conocimiento. La relación entre innovación y transformación productiva da cuenta de las condiciones socioeconómicas y culturales en las cuales se producen e incorporan los aprendizajes constituidos en conocimiento tácito y explícito.

Adicionalmente, la transformación productiva exige capacidades tanto humanas como tecnológicas que lleven a la competitividad de los espacios productivos (Hausmann $\mathcal{E}$ Klinger, 2006). En el gráfico 2 se sintetizan las relaciones que permiten la orientación del fortalecimiento de procesos productivos con innovación. Se parte de la dotación de factores (tierra, capital y trabajo) donde la educación y la capacitación juegan un papel fundamental en la construcción de entornos más productivos. Trabajadores más capacitados generan mayores procesos de innovación; empresas con mayores innovaciones son más competitivas en mercados internacionales; empresas con mejores desempeños demandan mano de obra más calificada y con mejores remuneraciones; trabajadores con remuneraciones más altas y concienciados de la importancia de la inversión en capital humano invierten en ellos y en sus familias y crean encadenamientos que impactan en la competitividad de las regiones en las que son hospedadas estas empresas y en la calidad de vida de los habitantes que desarrollan sus actividades económicas.

La discusión sobre cómo la innovación puede favorecer a los territorios no es un tema reciente. Autores como Romer (1989) han afirmado que las regiones con mayores tasas de inversión en capital humano tienen tasas más altas de desarrollo, industrias más competitivas y trabajadores con mayores habilidades para la observación, análisis e implementación de procesos productivos.

Méndez (2002) enlista las características de los territorios innovadores y los efectos que tiene la innovación en los mismos, en otras palabras, rinde cuentas del círculo virtuoso de la innovación y el desarrollo territorial. 
Innovación empresarial: una mirada desde la competitividad, el desarrollo local y la transformación productiva ...

Gráfico 1. La innovación a partir del desarrollo local con competitividad y transformación productiva: de lo local a lo internacional.

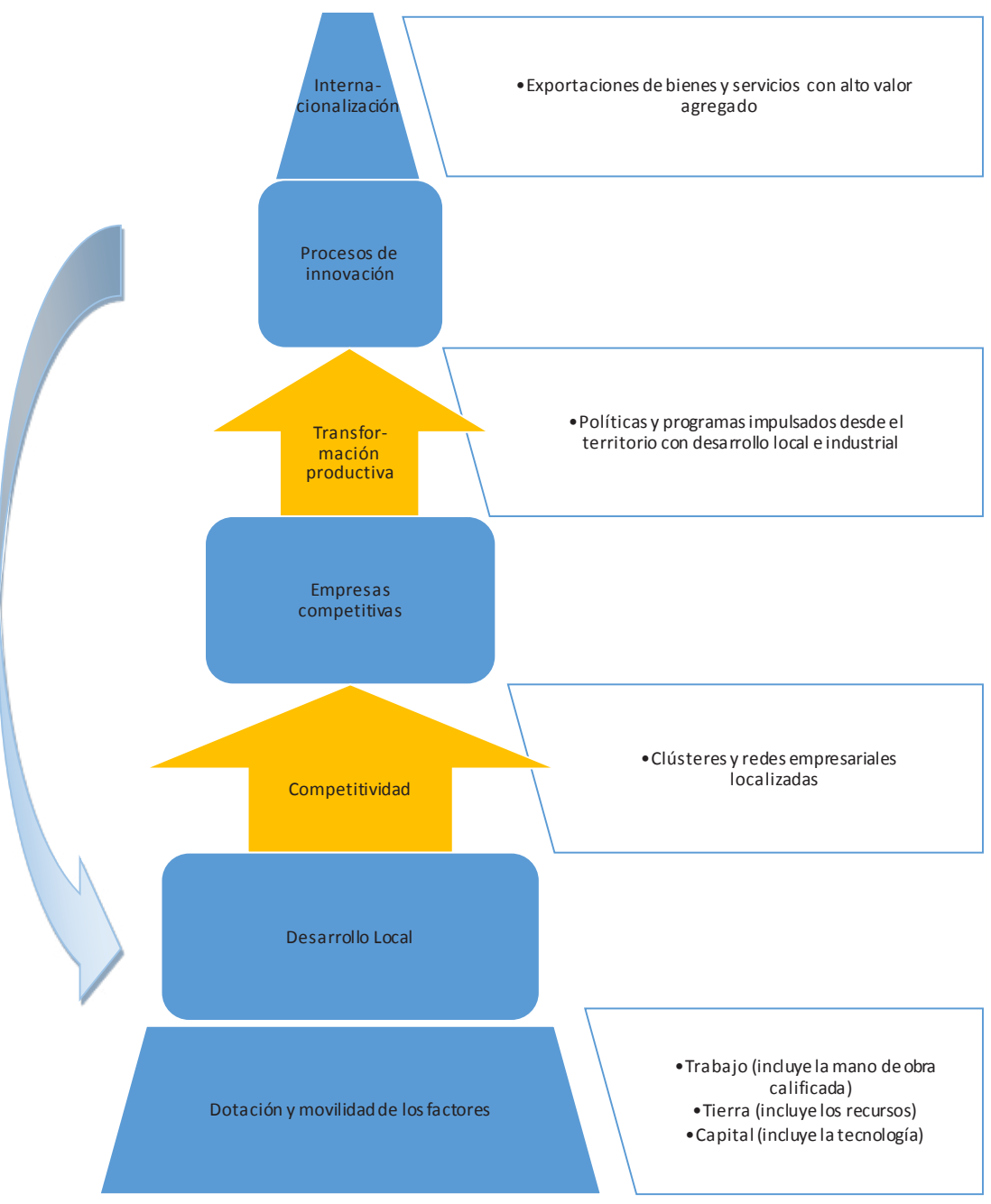

Fuente: elaboración propia

La innovación y el avance técnico son reconocidos como fuerzas fundamentales detrás de una amplia gama de fenómenos económicos como el crecimiento de la productividad, la competencia entre firmas en sectores como el electrónico y el farmacéutico, los patrones de comercio internacional en bienes manufacturados y muchos otros (Nelson \& Winter, 1982). Es decir, que la innovación ayuda a explicar la competitividad de las firmas. Y es así como Schumpeter $(1934,1950)$ lo plantea al darle un lugar central a la innovación dentro de su teoría del desarrollo económico. Es la innovación (las "nuevas combinaciones") la que permite que las firmas generen 
el desarrollo (el "cambio económico") a través de la superación de la competencia ("la eliminación competitiva de lo viejo") (Schumpeter, 1934). La innovación es una de las estrategias de las firmas al momento de competir, y como tal altera las condiciones del mercado a favor de aquellas firmas que pueden implementarla.

Gráfico 2. Territorios innovadores: características internas y principales efectos.

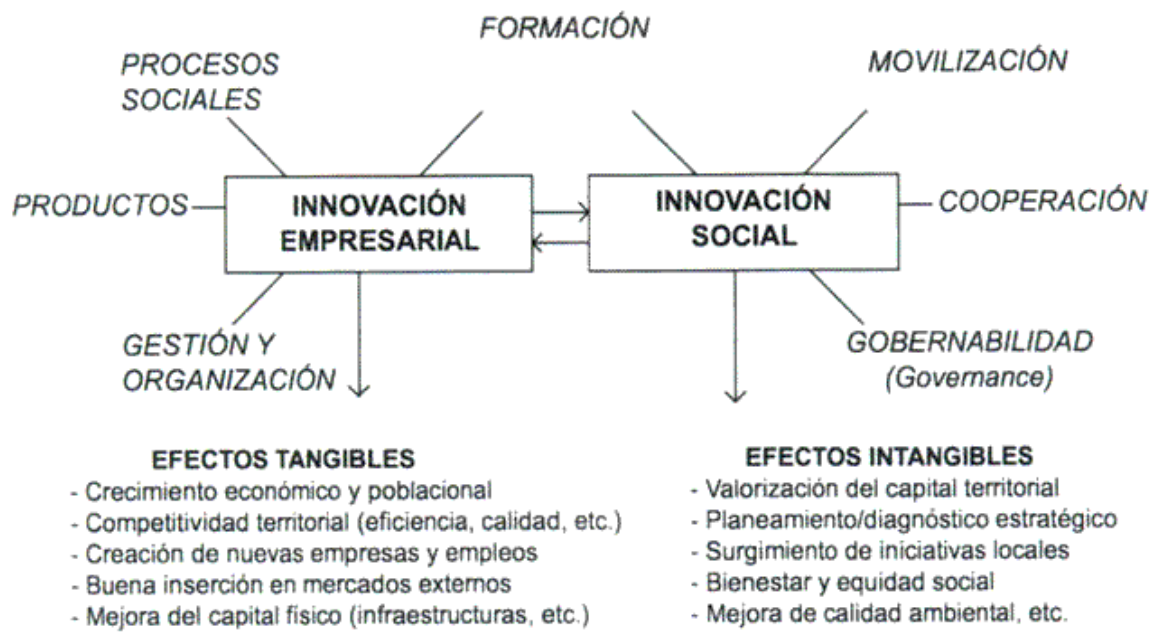

Fuente: Méndez (2002, p. 69)

La relación entre innovación y competitividad esta mediada también por los clústeres. Porter (1990) ha resaltado el importante papel que juegan para la competitividad nacional, estatal y local en la economía global. Los clústeres son concentraciones geográficas de empresas interconectadas, proveedores especializados, prestadores de servicios, empresas de sectores afines e instituciones asociadas (universidades, agencias reguladoras, asociaciones comerciales, etc.). A estos actores los unen elementos comunes y complementarios. Más que sectores económicos individuales, los clústeres abarcan una gran variedad de industrias vinculadas y otras entidades relevantes para la competencia y la cooperación (Porter, 2000, p. 15).

La participación en un clúster ofrece muchas ventajas potenciales (y algunos riesgos) para la innovación y la reconversión industrial en comparación con una ubicación aislada. Las firmas en un clúster son, con frecuencia, capaces de percibir con mayor claridad y rapidez las necesidades de los clientes. Las firmas en un clúster se benefician de la concentración de firmas que poseen conocimiento y relaciones con los compradores, de la yuxtaposición de las firmas en industrias relacionadas, de la concentración de entidades generadoras de información especializada y de la sofisticación de los compradores. Las firmas en clúster frecuentemente pueden 
Innovación empresarial: una mirada desde la competitividad, el desarrollo local y la transformación productiva ...

discernir las tendencias de los compradores con mayor rapidez que su competencia aislada (Porter, 2000, p. 23).

La participación en clústeres también ofrece ventajas al momento de descubrir posibilidades de nuevas tecnologías, operación o entrega. Los participantes pueden tener una posición privilegiada para percibir la evolución de la tecnología, la disponibilidad de componentes y maquinaria, los conceptos de servicio y mercadeo, etc. Las relaciones existentes con otras entidades en el clúster (incluyendo universidades) facilitan tal aprendizaje, así como las visitas físicas y contactos cara a cara. Se facilita la observación directa de otras firmas. La firma aislada, por contraste, enfrenta costos más altos y más grandes impedimentos para combinar ideas y mayor necesidad de crear conocimiento en su interior (Porter, 2000, p. 23).

\section{RESULTADOS ${ }^{1}$}

La internacionalización con innovación es un proceso en construcción. Los resultados que se presentan en esta sección se obtuvieron a partir de entrevistas semiestructuradas a profundidad, entre junio y diciembre del 2011. El análisis de los datos se hizo a partir de la realización de una matriz con las categorías por las que se indagó y las percepciones de los empresarios al respecto.

La recolección de datos se realizó mediante entrevistas y observación de la planta física de las empresas. Las preguntas que guiaron el trabajo de campo estuvieron orientadas a los procesos de innovación, transferencia, apropiación de las TIC, cadenas productivas y competitividad (ver tabla 1)

Los casos seleccionados fueron ocho empresas pertenecientes a sectores de manufactura (4), alimentos (2) e importadoras (2). Cuentan todas con menos de 50 empleados, y siete de ellas se fundaron con posterioridad a la apertura económica; entre tanto, una de ellas fue creada antes de la década de 1990. Todas cuentan con $100 \%$ de capital nacional y tienen activos entre los $\$ 167$ y $\$ 4.980$ millones de pesos colombianos.

Las empresas consultadas en su mayoría surgieron en el período 2001-2011, son medianas, con tecnología y de origen nacional. El surgimiento se dio para mercados nacionales y de producción tradicional. El financiamiento de la empresa se inicia con ahorro familiar.

Los resultados presentados en este aparte se rigen por el artículo 4․, Ley 1581/ 2012 "Los datos suministrados serán tratados bajo los principios de legalidad, finalidad, libertad, veracidad, transparencia, acceso y circulación restringida, seguridad y confidencialidad". En ese sentido, los nombres de las empresas entrevistadas son protegidos. Para identificar a los entrevistados se asignan la letra A seguida de un número consecutivo. 
Marleny Cardona Acevedo - Suelen Castiblanco Moreno - Hugo Díaz Sánchez

Tabla 1.Perfil de las empresas entrevistadas

\begin{tabular}{|c|c|c|}
\hline Característica & $n=8$ & Porcentaje de participación \\
\hline $\begin{array}{l}\text { Año de creación } \\
1980-1990 \\
1991-2000 \\
2001-2011\end{array}$ & $\begin{array}{l}1 \\
2 \\
5\end{array}$ & $\begin{array}{c}12,5 \% \\
25 \% \\
62,5 \%\end{array}$ \\
\hline $\begin{array}{l}\text { Estructura de propiedad } \\
\text { Sociedad anónima simplificada (SAS) } \\
\text { Limitada }\end{array}$ & $\begin{array}{l}3 \\
5\end{array}$ & $\begin{array}{l}37,5 \% \\
62,5 \%\end{array}$ \\
\hline $\begin{array}{l}\text { Sector } \\
\text { Manufactura } \\
\text { Alimentos } \\
\text { Importadora }\end{array}$ & $\begin{array}{l}4 \\
2 \\
2\end{array}$ & $\begin{array}{l}50 \% \\
25 \% \\
25 \% \\
\end{array}$ \\
\hline $\begin{array}{l}\text { Número de empleados } \\
1-10 \\
11-50\end{array}$ & $\begin{array}{l}3 \\
5\end{array}$ & $\begin{array}{l}37,5 \% \\
62,5 \% \\
\end{array}$ \\
\hline $\begin{array}{l}\text { Intensidad } \\
\text { Capital } \\
\text { Trabajo }\end{array}$ & $\begin{array}{l}5 \\
3 \\
\end{array}$ & $\begin{array}{l}62,5 \% \\
37,5 \% \\
\end{array}$ \\
\hline $\begin{array}{l}\text { Rango activos } \\
\text { Más de } \$ 166.000 .000 \\
\$ 166.000 .001-\$ 1.661 .000 .000 \\
\$ 1.661 .000 .001-\$ 4.980 .000 .000\end{array}$ & $\begin{array}{l}2 \\
5 \\
1\end{array}$ & $\begin{array}{c}25 \% \\
62,5 \% \\
12,5 \%\end{array}$ \\
\hline $\begin{array}{l}\text { Origen del capital } \\
\text { Nacional } \\
\text { Extranjero }\end{array}$ & $\begin{array}{l}7 \\
1 \\
\end{array}$ & $\begin{array}{l}87,5 \% \\
12,5 \% \\
\end{array}$ \\
\hline $\begin{array}{l}\text { Tipo de producción } \\
\text { Producción tradicional } \\
\text { Maquila } \\
\text { Importación }\end{array}$ & $\begin{array}{l}3 \\
1 \\
4\end{array}$ & $\begin{array}{c}37,5 \% \\
12,5 \% \\
50 \% \\
\end{array}$ \\
\hline $\begin{array}{l}\text { Tipo de financiamiento } \\
\text { Ahorro familiar } \\
\text { Crédito formal } \\
\text { Otro }\end{array}$ & $\begin{array}{l}4 \\
1 \\
3\end{array}$ & $\begin{array}{c}50 \% \\
12,5 \% \\
37,5 \%\end{array}$ \\
\hline
\end{tabular}

Fuente: elaboración propia

\subsection{Desarrollo local: cadenas y redes}

En la consulta a empresarios se reconocen relaciones con otras empresas, pero no se encontró que los acuerdos de colaboración con otras empresas fueran parte de los programas de crecimiento de las mismas. 
Innovación empresarial: una mirada desde la competitividad, el desarrollo local y la transformación productiva ...

En la entrevista los empresarios afirmaron que los únicos acuerdos de colaboración que poseen son con sus clientes y proveedores. Reconocen la importancia, pero la falta de asociatividad se explica por las percepciones sobre los otros empresarios que están centradas en la desconfianza y en el exceso de 'burocracia' y requerimientos en las instituciones para acceder a redes del sector y falta de resultados visibles de la gestión de las redes. "Yo asistí con frecuencia a reuniones de Proexport y de la Cámara de Comercio de Bogotá. Al principio lo ofrecido suena muy útil pero se hacían reuniones y nada se concretaba, al final dejé de ir. Prefiero invertir ese tiempo en la empresa" (A6).

La apuesta por la construcción de redes implica el establecimiento de grupos con intereses comunes, que buscan la transformación de procesos, la creación y fortalecimiento de relaciones sociales inter e intra institucional, por medio de la articulación, comunicación y apoyo (de tipo financiero, capacitación para el recurso humano, dotación en infraestructura, recursos tecnológicos), los diversos actores del desarrollo local como son los agentes de administración central, regional, municipal, las universidades, las instituciones y los empresarios. Se busca el fortalecimiento y la construcción de redes que permitan dinamizar los procesos de emprendimiento empresarial impulsados para la generación de empleo y la creación de empresas.

\subsection{Transformación productiva}

La adquisición de nueva maquinaria se da en la mayoría de casos mediante la compra directa y los créditos formales. Los empresarios se enteran de las nuevas tecnologías mediante ferias, exposiciones, consultas en Internet, y en algunos casos por empresas de asesoría comercial. Las principales dificultades asociadas a la compra de maquinaria son la escasa información acerca de la maquinaria de punta en los diferentes sectores y la financiación de las máquinas. En algunos casos, la regulación en materia de importaciones es un obstáculo para la adquisición de tecnología. "En diversas ocasiones han dejado nuestra mercancía en puerto hasta dos meses con diferentes excusas. La única solución ha sido darles dinero a las personas de puerto para que dejen mover la maquinaria" (A6).

El acceso a las tecnologías de información y comunicación (TIC) ha mejorado la comunicación con clientes y proveedores y el acceso a información, de acuerdo con las percepciones de los empresarios. La herramienta tecnológica más utilizada es el teléfono (celular y fijo) y el computador, aunque en su mayoría los empresarios manifestaron preferir la comunicación cara a cara para la definición de contratos de compra o venta. 


\subsection{Competitividad}

Los empresarios entrevistados afirmaron que las principales dificultades asociadas a mejoras en la competitividad están relacionadas con problemas con los trámites aduanales, autoridades y transporte transfronterizo. Solo en un caso se mencionó como principal problema la falta de crédito para la adquisición de maquinaria.

Las respuestas de las empresas frente a presiones competitivas están asociadas, en orden de importancia, con la reducción de los ciclos de fabricación y entrega del producto, reducción de costos, generación de valor agregado mediante el uso de mejores etiquetas, empaques, entre otros, y la reducción del número de proveedores para eliminar tiempos de entrega de los insumos y costos.

En su mayoría, los empresarios entrevistados afirmaron tener proyectos a largo plazo para la planta. La introducción de nuevos productos, expansión de la capacidad instalada y establecimiento de alianzas con productores extranjeros son los principales referentes de las empresas entrevistadas para su actuación en los próximos años.

\subsection{Innovación}

Los resultados de la consulta a empresarios se centran en los procesos de innovación, transferencia de tecnología y apropiación de las TIC con internacionalización. Los entrevistados manifestaron que sus empresas atraviesan por procesos de innovación y transferencia tecnológica cada 1.5 años en promedio. Las innovaciones se centran en la introducción de nuevos productos, mejoras tecnológicas en el proceso de producción y la apropiación de tecnologías existentes. En el caso de empresas con casa matriz, las innovaciones en las empresas se han presentado por la eliminación de una línea de producto o por el cierre de la compañía. "Cuando la casa matriz nos dijo que dejaría de fabricar el producto que más vendíamos decidimos viajar a la planta, aprender del proceso de producción y fabricarlo aquí en nuestra planta. Hoy es la línea de producto líder de la empresa" (A3).

Los procesos de innovación están motivados por el aumento de la calidad de los productos con miras a su acceso a los mercados internacionales. "Si no hago transformación tecnológica, me quiebro" (A5). Los empresarios manifestaron que adicional al aumento de la calidad, la transformación tecnológica les permite conquistar y aumentar la participación en los mercados y disminuir costos en los procesos de producción.

Los efectos sobre el empleo de los procesos de innovación dependen de forma directa de la cualificación de los empleados. Los empresarios en su mayoría, 
afirmaron que la mano de obra no calificada disminuyó después de la transferencia tecnológica debido a la eliminación de procesos manuales. La demanda por mano de obra calificada aumentó por la necesidad de técnicos para el manejo de la maquinaria implementada y por la necesidad del desarrollo de nuevos procesos productivos. "Muchos empleados temían que trajéramos nuevas máquinas porque pensaban que perderían su empleo, los capacitamos en el manejo de las máquinas nuevas y les dimos tiempo, los que no pudieron adaptarse tuvieron que irse" (A3).

\section{DISCUSIÓN}

La transformación productiva es el resultado de la dinámica entre el capital humano y los recursos productivos en el territorio. La transformación productiva se potencia en los procesos de industrialización que generan competencias que se traducen en innovación. Es esta última la que posibilita competir con éxito en los mercados internacionales.

De acuerdo con Vásquez Barquero (1999, p. 31) y como se muestra en el gráfico 3, los procesos de industrialización en el marco del desarrollo local se caracterizan por la integración del sistema productivo en la sociedad. Las empresas colaboran entre ellas para conseguir economías de escala que les permitan ser competitivas y que no conseguirían de manera aislada. Así se crea un "entorno económico e institucional que provee a las empresas locales con recursos, servicios y redes de cooperación entre los actores que les permiten mejorar la competitividad en los mercados nacionales e internacionales".

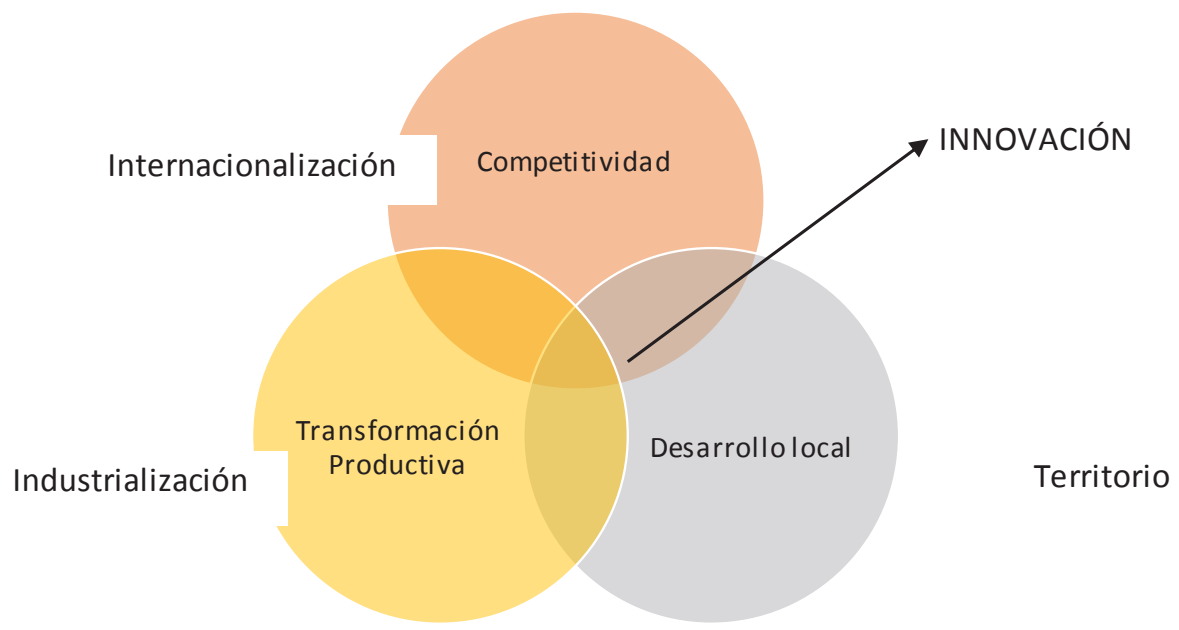

Gráfico 3. Dimensiones en la innovación

Fuente: elaboración propia 
Las implicaciones de estos resultados para las categorías estudiadas se presentan a continuación.

\subsection{Desarrollo local}

Las redes empresariales promueven la movilidad de tecnologías, distribuyen el conocimiento y elevan la productividad; por eso cuando se constituyen como parte de la práctica social son determinantes de la innovación (Szarka, 1996).

El proceso de internacionalización involucra en su quehacer dos elementos fundamentales: la apropiación del conocimiento generado en la economía y la posibilidad de trasladar esos aprendizajes a la transformación productiva de los procesos para ser más competitivos y productivos.

La capacidad de crear e incorporar conocimiento mediante la innovación, parte de la construcción social realizada entre agentes públicos, privados y la sociedad en general, que a través del desarrollo de políticas públicas de orden productivo y social llevan a las sociedades y a las empresas a insertarse de manera exitosa en contextos globales.

Cardona y Escobar (2012) aseguran que la producción es el espacio en el cual se muestran los resultados de las capacidades humanas y técnicas. La posibilidad de socializar los procesos productivos en el interior y exterior de las empresas es el eje central del fenómeno de difuminación o 'spillover' como mecanismo fundamental para la incorporación de la innovación.

Estos procesos de generación de conocimiento se enmarcan en los territorios y se alimentan de sus características culturales y sociales. Por esta razón, el análisis de la internacionalización y su relación con el desarrollo local se vuelve central. Sassen (2007) asegura que las regiones deben consolidarse a través de la combinación de factores productivos, conocimiento y empresas que les permita ser competitivas en escenarios globales.

Para Osorio (2010) la apuesta por la internacionalización requiere estrategias que provengan de los territorios, potencien las infraestructuras económicas de los mismos y respalden las iniciativas de desarrollo local. También es fundamental que las políticas de 'arriba hacia abajo' impulsen el compromiso de los actores gubernamentales y privados con los planes regionales, de forma tal que el éxito de la industria se traduzca en el crecimiento de los territorios. Finalmente, es fundamental la relación Universidad-Empresa-Estado que genere innovación con apropiación social. 
Innovación empresarial: una mirada desde la competitividad, el desarrollo local y la transformación productiva ...

Los avances de décadas recientes liderados por la Nueva Geografía Económica (NGE) ofrecen mayores posibilidades de estudiar los vínculos entre el desarrollo local, la competitividad y la transformación productiva. Para Krugman (1995) la habilidad de la NGE para expresar teorías del alto desarrollo con técnicas de modelización ortodoxas se convierte en una gran herramienta para el estudio del desarrollo, la geografía y la empresa y, por consiguiente, el próximo paso, en el estudio de la relación entre competitividad, desarrollo local, transformación productiva e innovación.

No obstante, como señala Foray (2004) es fundamental no dejar de lado que no todos los procesos de innovación y de spillovers (derramamientos) se pueden explicar exclusivamente desde la geografía. En primer lugar, los factores políticos pueden, en ocasiones, tener muy poco que ver con los efectos espaciales y mucha relación con la innovación; en segundo lugar, el potencial de las Tecnologías de la Información y la Comunicación (TIC) para reducir las restricciones espaciales debe incorporarse al análisis; y en tercer lugar, debe asumirse que la proximidad espacial no es el factor determinante, sino la manera en que las comunidades la utilizan para combinar sus activos tangibles e intangibles; es esta última característica la que hace que la proximidad pase de ser un fenómeno puramente geográfico, a una estructura organizacional efectiva para la creación de conocimiento.

\subsection{Transformación productiva}

La innovación es parte de la construcción social del tejido productivo; a la vez, es el resultado de la articulación entre capacidades humanas y desarrollo de productos que van a participar de los mercados. Es por eso que la internacionalización de la economía colombiana desde los procesos productivos, en el nuevo siglo, incorpora políticas de competitividad diseñadas desde la transformación productiva con innovación, fortalecimiento de capacidades y el desempeño empresarial (Cardona y Escobar, 2012).

Hausmann \& Klinger (2006) resaltan la necesidad de capacidades humanas e institucionales para el desarrollo productivo con transformación resultado de relaciones inter e intra empresariales en la localidad y entre países. Existen ventajas para la innovación asociadas a que las empresas o las personas logran agruparse en espacios productivos, en términos de intercambios de capacidades y tecnologías que llevan a ganancias por la aglomeración de agentes económicos en el territorio y en el intercambio de los factores influencian las decisiones sobre las que se implementarán o no las estrategias de las empresas. En este sentido existe un carácter social de la innovación. Este hace que la transformación productiva tenga un efecto 
público y privado, que se traduce en incrementos de productividad y competitividad con un ahorro de costes para las empresas y la sociedad.

La capacidad de innovación basada en la experiencia acumulada desde: 1) relaciones que tejen acuerdos desde la experiencia cruzada entre productores; 2) entre productores y clientes; y 3) entre clientes por intercambio de información; garantizan el incremento de la productividad y la competitividad y son resultado de condiciones socioeconómicas y culturales en las cuales se produce e incorpora el conocimiento.

\subsection{Competitividad}

La competitividad incluye la innovación como resultado de la incorporación de las capacidades humanas junto a las capacidades productivas y organizacionales para el logro de la transformación productiva; define los procesos y mecanismos que garantizan la eficiencia, eficacia y efectividad.

El desarrollo de cultura competitiva en las localidades incluye la innovación y tiene como antecedente potenciar las capacidades de creación. El diseño de políticas educativas para el trabajo, desde esta orientación, sustentadas además en el derecho al desarrollo, garantiza que los países y, en particular, las organizaciones tengan ventaja competitiva sostenida, desde el uso de los recursos que obtienen de su entorno. Así, las capacidades humanas hacen que se supere que "el solo hecho de que un país tenga una ventaja competitiva en una industria no implica que las empresas en esa industria sean competitivas en el nivel internacional, ni implica que el país sea competitivo" (Smith, 1994, p.122-123).

En palabras de Jorge Iván González (2011, p. 13)

[...] La innovación no se consigue únicamente con la inversión en ciencia y tecnología. La política pública debe facilitar la creación de empresa y, sobre todo, debe crear las condiciones que faciliten la interacción entre ciencia, tecnología y explotación comercial. Los márgenes de acción son importantes porque el mercado es muy imperfecto. En la secuencia de vida y muerte de las empresas, juega un papel central el espíritu empresarial del que habla Schumpeter en 1935 (como se cita en González, Jorge Iván, 2011). El buen empresario tiene una intuición y una capacidad subjetiva que le permite aprovechar las oportunidades del mercado.

Por último, la innovación es un esfuerzo desde la cultura, la economía, y la política para ganar en desarrollo y competitividad en las empresas, las regiones y los países. Las relaciones entre competitividad, desarrollo local y transformación productiva en el marco de la internacionalización son circulares y se refuerzan mutuamente con la presencia de la innovación. 


\section{CONCLUSIONES}

En este documento, teóricamente, se han expuesto las relaciones entre el desarrollo local, la competitividad, la transformación productiva y la innovación en el marco de la internacionalización. Una política hacia el exterior que apueste por iniciativas de abajo hacia arriba, con participación de la población, genera mayores niveles de apropiación de la tecnología, más capacidad para innovar y territorios competitivos que se insertan de manera más exitosa en los mercados internacionales.

El papel de lo local en el marco de la globalización contemporánea toma renovada vigencia en la discusión sobre la internacionalización de los territorios como alternativa para el desarrollo. El cómo y cuándo insertarse en la economía mundial, así como los efectos benéficos de hacerlo, son el eje central de los estudios de la glocalización y de la producción flexible.

A su vez, la generación de innovación requiere inversión en ciencia y tecnología por parte de las empresas y las instituciones, al ser el resultado de dinámicas empresariales e institucionales. Además, la internacionalización se constituye en un factor que estimula la innovación siempre y cuando se den condiciones desde el territorio que apoyen y sustenten los esfuerzos de los empresarios por penetrar nuevos mercados, como lo son las políticas sectoriales y los programas de acompañamiento. Sin embargo, los empresarios expresaron la limitada utilidad de estos programas para sus experiencias individuales, debido a los altos costos de transacción asociados a su funcionamiento y a la participación en los mismos.

La organización de la firma, la estructura y la innovación tecnológica fortalecen las dinámicas formales e informales en el territorio y son resultados de vínculos sociales asociados a procesos productivos. No obstante, los resultados del estudio mostraron que incluso los empresarios consideran los esfuerzos individuales como subóptimos frente a la posible utilidad derivada de la colaboración a través de redes y trabajo colectivo. Esta conciencia del valor de la cooperación no se materializa en mayor asociatividad, lo que plantea un espacio para futuras investigaciones.

Las estructuras institucionales también influyen en el sistema de causación circular alrededor de la innovación. En este contexto las políticas sectoriales son fundamentales para impulsar la transformación productiva. La posibilidad de competir con productos de talla mundial en los grandes mercados globales, tal como se ha planteado en la política de transformación productiva en el país, está atravesada por la capacidad de los actores públicos y privados en el territorio de crear el tejido que permite convertir las iniciativas individuales en tendencias colectivas. Sin esta, el valor agregado de las exportaciones del país permanece estructural- 
mente bajo y prevalece la internacionalización de productos primarios con baja o nula transformación. Los resultados de esta investigación sugieren un gran vacío entre la formulación de la política y la percepción de los empresarios respecto al acompañamiento del Estado.

Finalmente, se puede concluir que la innovación es una apuesta por la competitividad, desde el territorio, que tiene en cuenta la transformación productiva como la vía que garantiza el desarrollo empresarial. El éxito o fracaso de su incorporación en los procesos de internacionalización está íntimamente ligado con su capacidad de rendir cuentas de todas las dimensiones que están tras su generación.

\section{BIBLIOGRAFÍA}

Acs, Z. J. (2002). Innovation and the growth of cities. Massachusetts: Edward Eolgar Publishing Inc.

Acs, Z. J., \& Amorós, A. (2008). Entrepreneurship and Competitiveness Dynamics inLatin America. JENA ECONOMIC RESEARCH PAPERS, (059).

Cardona Acevedo, M., Cano Gambo Carlos., Osorio García Ana. (2005) Ciclo de vida y localización de las firmas en Colombia: Algunos elementos para la conceptualización. En: Revista Universidad EAFIT 41 (137). Pp. 44-59

Cardona Acevedo, M., Escobar Acevedo, S. (2012). Innovación en la transformación productiva industrial: aportes a la discusión. En: Semestre Económico. 15 (31). Pp. 12-151.

Cerrato, D. \& Depperu, D. (2011). Unbundling the construct of firm-level international competitiveness. The Multinational Business Review (19) 4. 311-331

Dahlman, C E Frischtak, C (1993) National Innovation Systems. A Comparative Analysis. Richard Nelson, Eds. Oxford University Press. New York.

Foray, D (2004) La economía del conocimiento. Massachusetts Institute of Technology. Cambridge.

Garay, L (2004) Colombia: estructura industrial e internacionalización 1967-1996. Biblioteca virtual del Banco de la República. Colombia: estructura industrial e internacionalización 1967-1996.

González, J. (2011). Psicología, biología y economía. En: Suma de Negocios, 2(2). Pp. 7-16.

Hausmann, R., E Klinger, B. (2006). Structural transformation and patterns of comparative advantage in the product space. CID Working Paper, (128).

Méndez, R (2002) Innovación y desarrollo territorial: algunos debates teóricos recientes. Revista Eure XXVIII (84) pp. 63-83.

Krugman, P. (1995). Desarrollo, geografía y teoría económica. Barcelona, España: Antonio Bosch Editor.

OCDE (2005). Manual de Oslo: Guía para la recogida e interpretación de datos sobre innovación. París, Grupo Tragsa (3 ed.), 188 pp. 
Innovación empresarial: una mirada desde la competitividad, el desarrollo local y la transformación productiva ...

Osorio, D (2010) ¿̇Internacionalización con o sin desarrollo local?: Un asunto de estrategia. Suma de Negocios. Vol. 1 (2)

Porter, M. (1990). The competitive advantage of nations. New York: Free Press.

Porter, M (2000) Location, competition, and economic development: Local clusters in a global economy. Economic development quarterly. 14 (1), pp. 15-34.

Robledo, J., Gómez, F. A., E Restrepo, J. F. (2008). Relación entre capacidades de innovación tecnológica y desempeño empresarial en Colombia. In Memorias del Primer Congreso Internacional de Gestión Tecnológica e Innovación (Vol. 21).

Romer, P. (1989) Human capital and growth: Theory and evidence. New working paper series. Working papers N. ${ }^{\circ}$ 3173. National Bureau of Economic Research (NBER). Cambridge.

Sassen, S. (2007) El reposicionamiento de las ciudades y regiones urbanas en una economía global: ampliando las opciones de políticas y gobernanza. Revista Eure (Vol. XXXIII, N. ${ }^{\circ}$ 100) Santiago de Chile. Diciembre, Pp. 9-34.

Schumpeter, J. A. (1934). The Theory of Economic Development. Cambridge, Mass.: Harvard University Press.

Schumpeter, J. A. (1935). The Analysis of Economic Change. Review of Economic Statistics. 17(4), Pp. 2-10

Schumpeter, J. A. (1950). Capitalism, Socialism, and Democracy, 3rd ed. New York: Harper.

Smith, A (1994). La riqueza de las naciones. Madrid, España: Alianza Editorial. 808 pp.

Szarka, J. (1996) "Las redes y la pequeña empresa". En: Desarrollo y Gestión de MES: aportes para un debate necesario. Universidad Nacional General de Sarmiento.

Vásquez Barquero, A. (1999) Desarrollo, redes e innovación. Lecciones sobre desarrollo endógeno. Madrid, España: Ediciones Pirámide.

Vásquez Barquero, A. (2009) Desarrollo local, una estrategia para tiempos de crisis. Universitas Forum. 1 (2) 
